\title{
AN EFFICIENT SYNTHETIC METHOD FOR THE SYNTHESIS OF NOVEL CHIRAL ANALOGOUS OF CILAZAPRIL AND CCKA ANTAGONISTS
}

\author{
H. Ali Döndaş* \\ Mersin University, Faculty of Pharmacy, Yenisehir Campus,33342 Mersin-Turkey
}

\begin{abstract}
An efficient synthetic method for the synthesis of novel chiral cilazapril and analogous of CCKA_antagonists is desciribed. The reactions proceed $v i a$ acidic deprotection of tert-butyl -9 (S)-phthalimido octahydro-6, 10-dioxo- -6Hpyridazo[1,2-a] [1,2] diazepine-1(S)-carboxylate, without isolation, which then coupled with 1-methyl-3-amino-1,3dihydro-5-phenyl-(2H)-1,4-benzodiazepine-2-one and allylamine using diethyl phosphorocyanidate as a coupling reagent afforded novel peptidomimetics in excellent yield. This sequence of reactions provided a simple four-reaction sequence and efficient synthesis of novel peptidomimetics.
\end{abstract}

Keywords: Cilazapril, A.C.E inhibitors, cholecystokinin, benzodiazepine

\section{Introduction}

Recently interest has turned to peptide molecules which can be as active and more stable than natural peptide $^{1}$ that are expected to have same therapeutic effects as natural peptide counterparts, with the added advantage of metabolic stability ${ }^{2}$. Angiotensin Converting Enzyme (A.C.E.) inhibitors such as enalapril (1) and cilazapril (2) are effective in the treatment of essential hypertension ${ }^{3}$. A.C.E (E.C. 3.4.15.1) has assumed increasing importance in the therapy of hypertension of congestive heart failure ${ }^{4}$. Cilazapril $(2)^{3,5,6}$ is one of the most potent of the new generation of antihypertensive drugs. It is an inert prodrug with a high degree of oral bioavaibility and is the monoethyl ester prodrug form of a potent, specific, long-acting antihypertensive inhibitor of Angiotensin Converting Enzyme (A.C.E.). The biochemical and pharmacological properties of this compound has been found most potent and longest - acting compared with those of parent drug enalaptril $(1)^{5}$. The dicarboxylic acid $(2, \mathrm{R}=\mathrm{H})$ is an active A.C.E. inhibitor. The corresponding mono ester $(2, \mathrm{R}=\mathrm{Et}$, cilazapril) is used for in vivo inhibition of A.C.E. This prodrug has better biological characteristics as an antihypertensive in mammals than the corresponding dicarboxylic acid which is formed from cilazapril in vivo as a result of the action of esterases. ${ }^{6}$

A substantial amount of novel chemistry ${ }^{7-9}$, mainly targeted at the A.C.E inhibitors, has been developed from the parent 9-aminopyrdazino diazepine and t-butyl-9-amino-octahydro-6,10-dioxo-6H-pyridazino[1,2-a] $[1,2]$ diazepine-1-carboxylate and related bicyclic compounds. ${ }^{6.10}$ 
Benzodiazepines have been known to posses important medicinal properties for the last several years. Some benzodiazepines derivatives $(3,4)$ have been found to be potent antagonist of the peptide hormones cholecystokinin (CCK) and gastrin. ${ }^{11-14}$ The 1,4-benzodiazepines have constitutes a class of widely used anxiolytic and anticonvulsant drug. ${ }^{15}$ Recently some amino and succinoylamino benzodiazepines such as (3) have been useful in treatment of neurologic disorders related to $\beta$-amyloid production such as Alzheimer's disease and Down's Syndrome ${ }^{16}$.<smiles>CCOC(=O)C(CCc1ccccc1)CC(C)C(=O)N1CCCC1C(=O)O</smiles>

(1)<smiles>O=C(O)C(CCc1ccccc1)NC1CC=[N+]2CCCC(C(=O)O)N2C1C(=O)O</smiles>

(2) $\mathrm{R}=\mathrm{H}$ and $\mathrm{Et}$<smiles>CC(NC(=O)Cc1cc(F)cc(F)c1)C(=O)N[C@@H]1N=C(c2ccccc2)c2ccccc2N1C</smiles>

(3)<smiles>Cc1cccc(NC(=O)NC2N=C(c3ccccc3)c3ccccc3N2C)c1</smiles>

(4)

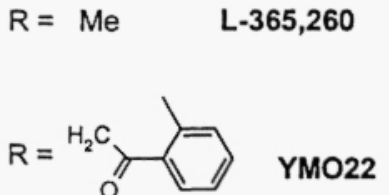

We have previously shown that amines of 1,4-benzodiazepine ${ }^{17,18}$ and 9-aminopyrdazino diazepine $e^{20,21}$ react with aldehydes to generate imines which then gave azomethine ylides via thermal and metal catalysed processes. 1,3-Dipolar cycloaddition reactions of which with chiral and achiral dipolarophiles afforded cycloadduct in good to excellent yield. ${ }^{17-22}$ Thus imine - azomethine ylide - cycloaddition cascade chemistry has provided a series of spirobenzodiazepines related to MK-329 $17-19$ and spirocilazapril analogues ${ }^{20 \cdot 22}$ and potentially offers a facile route conformationally restricted analogues of (2-4) ${ }^{17-22}$.

Results and Discussion: We now report a study of efficient synthetic method for the synthesis of novel chiral cilazapril and analogous of CCKA antagonists. The reaction proceeds via coupling reaction of tertbutyl-octahydro-6, 10-dioxo-9 (S)-phthalimido-6H-pyridazo[1,2-a] [1,2] diazepine-1(S)-carboxylate (5) with p-toluene sulphonic acid salt of 1-methyl-3-amino-1,3-dihidro-5-phenyl-(2H)-1,4-benzodiazepine-2-one (6) and allyl amine. This substrate allows the influence of the new stereocentre on the cascade to be assessed with respect to the configuration of (6). Thus reaction of (5) with (6) afforded compound (7) which then gave (8) via hyrazinolysis. The reactions proceed via acidic deprotection of tert-butyl moiety of (5) employing 
TFA(DCM, rt, 10h)) under an argon atmosphere afforded 9 (S)-phthalimido octahydro-6, 10-dioxo- -6Hpyridazo[1,2-a] [1,2] diazepine-1(S)-carboxylic acid. Without isolation, the acid was coupled with 1-methyl-3amino-1,3-dihidro-5-phenyl-(2H)-1,4-benzodiazepine-2-one using diethyl phosphorocyanidate (DEPC) as a coupling reagent in anhydrous DMF at $0{ }^{\circ} \mathrm{C}$ for $30 \mathrm{~min}$ and room temperature for $16 \mathrm{~h}$ gave (7). The free amine (8) of that was liberated from its phthalimido (7) by treatment with hydrazine hydrate and trituration filtration. The chiral compound (8) were obtained in $95 \%$ yield as a 1:1 mixture of diastereoisomer. The reaction proceeds under mild condition in excellent yield. This sequence of reactions provided a simple four reaction sequence and efficient synthesis of novel peptide molecules. In a similar manner as outlined in scheme 2 an example of $\mathrm{N}$-allyl substituted cilazapril analogous (10) were obtained from (5) in the presence of allylamine in $89 \%$ yield as a single stereoisomer. The structure of $(7-10)$ were assigned on the basis of ${ }^{1} \mathrm{H}$ and ${ }^{13} \mathrm{C}$ NMR spectra, ${ }^{2} \mathrm{D}$ COSY, DEPT and HETCOR studies (see experimental) and conforms to that previous studies. $^{17-22}$<smiles>CC(C)(C)OC(=O)C1CCCN2C(=O)CC[C@H](N3C(=O)c4ccccc4C3=O)C(=O)N12</smiles>

(5)<smiles></smiles>

(6)<smiles>[2H][C@@]1(NC(=O)[C@H]2CCCN3C(=O)CC[C@H](N)C(=O)N23)N=C(c2ccccc2)c2ccccc2N(C)C1=O</smiles>

(8)
Scheme 1<smiles>[2H][C@@]1(NC(=O)C2CCCN3C(=O)CC[C@H](N4C(=O)c5ccccc5C4=O)C(=O)N23)N=C(c2ccccc2)c2ccccc2N(C)C1=O</smiles>

TFA, DCM, rt, 10h

$\mathrm{Et}_{3} \mathrm{~N}, \mathrm{DEPC}$

DMF, ${ }^{\circ} \mathrm{C}->\mathrm{rt}, 16 \mathrm{~h}$

(7)<smiles>CCCCN[C@H]1N=C(c2ccccc2)c2ccccc2N(C)C1=O</smiles> 
(5)

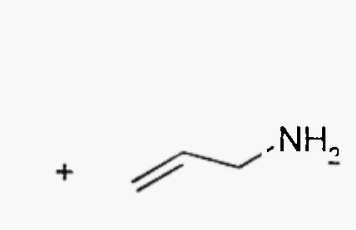

(9)

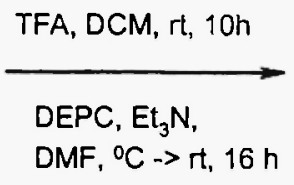

$D M F,{ }^{\circ} \mathrm{C} \rightarrow \mathrm{rt}, 16 \mathrm{~h}$

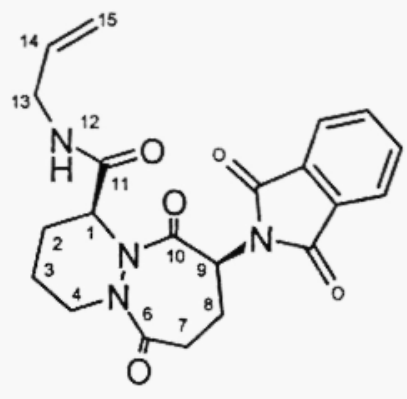

(10)

\section{Scheme 2}

In conclusion, an efficient method for the synthesis of novel chiral cilazapril and cholecystokinin analogues have been developed via a simple four-reaction sequence in one-pot reaction. Similar methodology should provide a general route to other biologically important cilazapril and benzodiazepine analogues.

Acknowledgements: The author thanks TUBITAK [(TBAG-2154 (102T033)] and Mersin University for support.

\section{Experimental}

Microanalyses were obtained using a Carlo - Erba Model 1106 instrument. Mass spectra were recorded at 70 ev on a VG Autospec mass spectrometer. Melting points were determined on a Kofler hot stage apparatus and are uncorrected. Nuclear magnetic resonance spectra and decoupling experiments were determined at 250 MHz. on a Q.E 250 instrument and at $500 \mathrm{MHz}$ on a Bruker AM500 spectrometer as specified. Chemical shifts are given in parts per million $(\delta)$ downfield from tetramethylsilane as internal standard. Spectra were determined in deuteriochloroform except where otherwise stated. The following abbreviations are used; $s=$ singlet, $d=$ doublet, $t=$ triplet, $q=$ quartet,$m=$ multiplet, $b r=$ broad and brs= bioad singlet. Flash column chromatography was performed using silica gel 60 (230-400 mesh). Kieselgel columns were packed with silica gel GF254 (Merck 7730). Petroleum ether refers the fraction with b.p $40-60^{\circ} \mathrm{C}$ unless otherwise specified. Optical rotations were dctermined on an Optical Activity Ltd., AA1000 polarimeter. TFA were purchased from Aldrich and used as received.

Compound 7. To a solution of (5) $(0.25 \mathrm{~g}, 0.583 \mathrm{mmol})$ in dichloromethane (DCM) $(20 \mathrm{ml})$ in $50 \mathrm{ml}$ roundbottom flask flushed with argon was added TFA $(2.25 \mathrm{ml})$. The mixture was stirred for $10 \mathrm{~h}$, the solvents were removed under reduced pressure, TFA was azeotropically removed with benzene and the resulting residue was dried in vacuo. To a suspension of the residue and salt of (6) $(0.382 \mathrm{~g}, 0.874 \mathrm{mmol})$ in $4 \mathrm{ml}$ of 
dimethylformamide(DMF) was added to a solution of diethyl phosphorocyanidate (DEPC) $(0.104 \mathrm{~g}, 0.641$ $\mathrm{mmol})$ in $4 \mathrm{ml}$ of DMF and $\mathrm{Et}_{3} \mathrm{~N}(0.294 \mathrm{~g}, 2.91 \mathrm{mmol})$ in $4 \mathrm{ml}$ of DMF. After the mixture were stirred at $0{ }^{\circ} \mathrm{C}$ for $30 \mathrm{~min}$ and room temperature for $16 \mathrm{~h}$, the solvent was removed in vacuo. Purification by colum chromatography $1 \% \mathrm{MeOH} / \mathrm{DCM}$ gave pale pink amorphous solid. mp $172-174{ }^{\circ} \mathrm{C}$. $[\alpha] \mathrm{D}=-98.3(\mathrm{c}, 0.1$, EtOH). HRMS (ES): Found 641.2115, $\mathrm{C}_{34} \mathrm{H}_{30} \mathrm{~N}_{6} \mathrm{O}_{6} \mathrm{Na}$ requires: 641.2125. $\mathrm{m} / \mathrm{z}$ (\%) (FAB): $6.19(\mathrm{M}+1,100)$, 298 (15), 249 (13), 221 (28), 186 (25). $\delta_{\mathrm{H}}\left(500 \mathrm{MHz}, \mathrm{CDCl}_{3}\right.$ ): 7.86 (m, 2H, ArH), 7.84 (m, 2H, ArH), $7.72-$ $7.62(\mathrm{~m}, 3 \mathrm{H}, \mathrm{ArH}$ and $\mathrm{NH}), 7.59-7.28(\mathrm{~m}, 7 \mathrm{H}, \mathrm{ArH}), 5.52-5.50(\mathrm{~m}, 2 \mathrm{H}, 1-\mathrm{H}$ and $1-\mathrm{H}), 5.33(\mathrm{~m}, 1 \mathrm{H}, 9-\mathrm{H}), 4.70$ (m, $1 \mathrm{H}, 4 \beta), 3.66(\mathrm{~m}, 1 \mathrm{H}, 7 \beta), 3.47$ (s, 3H, NMe), 3.40 (m, 1H, $8 \alpha), 3.0(\mathrm{~m}, 1 \mathrm{H}, 4 \alpha), 2.48-2.31$ (m, 3H, $7 \alpha$, $8 \beta, 2 \alpha), 2.04-1.96(\mathrm{~m}, 2 \mathrm{H}, 2 \beta$ and $3 \alpha), 1.78(\mathrm{~m}, 1 \mathrm{H}, 3 \beta)$.

Compound 8 was obtained (95\%) as a $1: 1$ mixture of diastereoisomer pale yellow prism, mp $128-131{ }^{\circ} \mathrm{C}$. $[\alpha]_{D}=-102.2$ (c, 0.1, EtOH). HRMS (ES): Found 511.2047, $\mathrm{C}_{26} \mathrm{H}_{28} \mathrm{~N}_{6} \mathrm{O}_{6} \mathrm{Na}$ requires: 511.2070. $\mathrm{m} / \mathrm{z}$ (\%)(FAB): 489 (M+1, 100), 311 (12), 266 (15), 249 (25), 221 (58), 168 (24), 149 (18). Found: C, 56.3; H, $5.85 ; \mathrm{N}, 15.55 ; \mathrm{C}_{26} \mathrm{H}_{28} \mathrm{~N}_{6} \mathrm{O}_{4} .4 \mathrm{H}_{2} \mathrm{O}$ required: $\mathrm{C}, 55.7 ; \mathrm{H}, 6.40 ; \mathrm{N}, 15.0 \% . \delta\left(250 \mathrm{MHz}, \mathrm{CDCl}_{3}\right): 7.89$ (br, $1 \mathrm{H}$, $\mathrm{CONH}), 7.58-7.55(\mathrm{~m}, 2 \mathrm{H}, \mathrm{ArH}), 7.52-7.25(\mathrm{~m}, 7 \mathrm{H}, \mathrm{ArH}), 5.42-5.30\left(\mathrm{~m}, 2 \mathrm{H}, 1-\mathrm{H}\right.$ and $\left.\mathrm{l}^{\prime}-\mathrm{H}\right), 4.58(\mathrm{~m}, 1 \mathrm{H}, 4 \beta)$, $3.81(\mathrm{~m}, 1 \mathrm{H}, 7 \beta), 3.79(\mathrm{~m}, 1 \mathrm{H}, 8 \alpha), 3.51$ (br, $\left.2 \mathrm{H}, \mathrm{NH}_{2}\right), 3.46$ and 3.44 (2xs, 3H, N-Me isomer), $2.94(\mathrm{~m}, 1 \mathrm{H}$, $4 \alpha$ ), $2.50(\mathrm{~m}, 1 \mathrm{H}, 7 \alpha), 2.25-2.20(\mathrm{~m}, 2 \mathrm{H}, 8 \beta$ and $2 \alpha), 2.10-1.77(\mathrm{~m}, 4 \mathrm{H}, 2 \beta, 3 \alpha, 3 \beta, 9 \alpha)$.

Compound (10). To a solution of (5) $(0.25 \mathrm{~g}, 0.583 \mathrm{mmol})$ in dichloromethane (DCM) $(15 \mathrm{ml})$ in $50 \mathrm{ml}$ round-bottom flask flushed with argon was added TFA $(4.5 \mathrm{ml})$. The mixture was stirred for $10 \mathrm{~h}$, the solvent were removed under reduced pressure. TFA was azeotropically removed with benzene and the resulting residue dried at $70^{\circ} \mathrm{C}$ in vacue. To a suspension of the residue and allyl amine (9) (0.033 g, 1 eq) in $3 \mathrm{ml}$ of DMF was added a solution of DEPC (diethyl phosphorocyanidate) $(0.104 \mathrm{~g}, 0.641 \mathrm{mmol})$ in $3 \mathrm{ml} \mathrm{DMF}$ and $\mathrm{Et}_{3} \mathrm{~N}(0.07 \mathrm{~g}, 1.2 \mathrm{eq})$ in $3 \mathrm{ml}$ of DMF. After the mixture were stirred at $0{ }^{\circ} \mathrm{C}$ for $30 \mathrm{~min}$ and room temperature for $6 \mathrm{~h}$, the solvent was removed in racuo. Purification by column chromatography $1 \% \mathrm{MeOH} / \mathrm{DCM}$ gave colourless prisms in $89 \%$ yield. mp $179-181{ }^{\circ} \mathrm{C} .[\alpha] \mathrm{D}^{=}-90.3$ (c, $\left.0.1, \mathrm{EtOH}\right)$. Found: C, 61.35; H, 5.5; N, 13.45; $\mathrm{C}_{21} \mathrm{H}_{22} \mathrm{~N}_{4} \mathrm{O}_{5}$ required: $\mathrm{C}, 61.45 ; \mathrm{H}, 5.35 ; \mathrm{N}, 13.65 \% . m / z(\%)(\mathrm{ES}): 433\left(\mathrm{M}^{+}+\mathrm{Na}, 100\right) . m / z(\%)(\mathrm{EI})$ : $411\left(\mathrm{M}^{+}+1,20\right), 355(50), 326(75), 298(98), 186(100), 104(27)$ and $85(57) .8 \mathrm{H}\left(500 \mathrm{MHz}, \mathrm{CDCl}_{3}\right) 7.90-$ $7.84(\mathrm{~m}, 2 \mathrm{H}, \mathrm{Ar}-\mathrm{H}), 7.86-7.84(\mathrm{~m}, 2 \mathrm{H}, \mathrm{Ar}-\mathrm{H}), 6.0(\mathrm{br}, 1 \mathrm{H}, \mathrm{NH}), 5.83\left(\mathrm{~m}, 1 \mathrm{H}, \mathrm{CH}=\mathrm{CH}_{2}\right), 5.26(\mathrm{t}, 1 \mathrm{H}, 1-$ $\mathrm{CHCONH}), 5.16-5.13\left(\mathrm{~m}, 2 \mathrm{H}, 15-\mathrm{CH}_{2}\right) 5.10(\mathrm{~m}, 1 \mathrm{H}, 9-\mathrm{CHN}), 4.67\left(\mathrm{~m}, 1 \mathrm{H}, 4-\mathrm{CH}_{2} \alpha\right), 3.95-3.90(\mathrm{~m}, 2 \mathrm{H}$, $\left.\underline{\mathrm{CH}_{2}} \mathrm{NH}\right) .3 .50\left(\mathrm{~m}, 1 \mathrm{H}, 2-\mathrm{CH}_{2}\right), 3.20\left(\mathrm{~m}, 1 \mathrm{H}, 7-\mathrm{CH}_{2}\right), 3.02\left(\mathrm{~m}, 1 \mathrm{H}, 4-\mathrm{CH}_{2} \beta\right), 2.60\left(\mathrm{~m}, 1 \mathrm{H}, 2\right.$ '- $\left.\mathrm{CH}_{2}\right), 2.56(\mathrm{~m}$, 
$\left.1 \mathrm{H}, 7^{\prime}-\mathrm{CH}_{2}\right), 2.11\left(\mathrm{~m}, 1 \mathrm{H}, 8-\mathrm{CH}_{2}\right), 2.05\left(\mathrm{~m}, 1 \mathrm{H}, 3-\mathrm{CH}_{2}\right), 1.98\left(\mathrm{~m}, 1 \mathrm{H}, 8^{\prime}-\mathrm{CH}_{2}\right), 1.88\left(\mathrm{~m}, 1 \mathrm{H}, 3^{\prime}-\mathrm{CH}_{2}\right) . \delta_{\mathrm{C}}(500$ $\left.\mathrm{MHz}, \mathrm{CDCl}_{3}\right)$ : $19.17\left(\mathrm{CH}_{2}\right), 24.49\left(\mathrm{CH}_{2}\right), 26.33\left(\mathrm{CH}_{2}\right), 30.38\left(\mathrm{CH}_{2}\right), 41.95\left(\mathrm{CH}_{2}\right), 42.03\left(\mathrm{CH}_{2}\right), 42.90(\mathrm{CH})$, $53.08(\mathrm{CH}), 117.09(\mathrm{CH}), 123.59(2 \mathrm{C}, \mathrm{CH}), 133.43(\mathrm{CH}), 134.33(2 \mathrm{C}, \mathrm{CH}), 167.96(2 \mathrm{C}, \mathrm{q}), 168.42(2 \mathrm{C}, \mathrm{q})$, $168.57(\mathrm{q}), 172.0(2 \mathrm{C}, \mathrm{q})$.

\section{References}

1. S. Hanessian, G. Mc-Naugton-Smith, H.G. Lombart, and W.D. Lubell, Tetrahedron, 53, 1278912854 (1997).

2. G.L. Olson, M. Voss, D.E. Hill, M. Kahn, V.S. Madison, C.M. Cook, J. Am.Chem. Soc., 112, 323$331(1990)$.

3. S.H. Gavra, H.R. Brunner, G.A. Turini, New. Engl J. Med., 298, 991-995 (1978); I.L. Natoff, M.R. Attwood, D.A. Eichler, P. Kogler, C.H. Kleinbloesem, P.V Brummelen, Cardiovascular Drug. Rew., 8, 1-24 (1990).

4. C.S. Sweet, D.M. Gross, P.T. Arbegast, J. Pharmacol Exp. Ther, 216, 558-566 (1981).

5. I.L. Natoff, J.S. Nixon, R.J Francis, L.R. Klevans, M. Brewster, J. Budd, A.T. Patel, J. Wenge, E. Worth, Journal of Cardioviscular Pharmacology, 7, 569-580 (1985).

6. R.M. Attwood, C.H. Hassill, A. Kröhn, G. Lawton, S. Redshaw, J. Chem. Soc. Perkin. Trans. I, 1011-1019 (1986).

7. R.M. Attwood, R.J. Francis, C.H. Hassall, A. Kröhn, G. Lawton, I.L. Natoff, J.S. Nixon, S. Redshaw, W.A. Thomas, FEBS Lett., 165, 201-206 (1984).

8. W.A. Thomas, P.J. Gilbert, J. Chem. Soc., Perkin Trans. II, 1077-1082 (1985).

9. C.H. Hassall, A. Kröhn, C.J. Moody and W.A. Thomas, J. Chem. Soc. Perkin Trans. I, 155-164 (1984).

10. W.A. Thomas and I.W.A. Whitcombe, J. Chem. Soc., Perkin Trans. II, 747-755 (1986).

11. (a). M.G. Bock, R.M. DiPardo, B.E. Evans, K.E. Rittle, W.L. Whittler, D.F. Veber, P.S. Anderson, and R.M. Freidinger, J. Med. Chem., 32, 13-16 (1989). (b). V.J. Lotti, R.S.L. Chang, Eur. J. Pharmacol., 162, 273 (1989). (c) M.G. Bock, R.M. DiPardo, B.E. Evans, K.E. Rittle, W.L. Whittler, V.M. Garsky, K.I. Gilbert, J.L. Leighton, K.L. Carson, E.V. Mellin, D.F. Veber, R.S.L. Chang, V.J. Lotti, S.B. Freedman, A.J. Smith, S. Patel, P.S. Anderson, R.M. Friedinger, J. Med.Chem, 36, 4276-4292 (1993). 
12. A. Nishida, K. Miyata, R. Tsutsumi, H. Yuki, S. Akuzawa, A. Kobayashi, T. Kamato, H. Ito, M. Yamano, Y. Katuyama, M. Satoh, M. Ohta, K. Honda, J. Pharmacol. Exp. Ther., 269, 725-731 (1994).

13. R.S.L Chang, V.J. Lotti, R.L. Monaghan, J. Bimbaum, E.O. Stapley, M.A. Goetz, G. AlbersSchonberg, A.A. Paichett, J.M. Liesch, O.D. Hensens, and J.P. Spinger, Science, 230, 177179 (1985).

14. B.E. Evans, M.G. Bock, K.E. Rittle, R.M. DiPardo, W.L. Whittler, D.F. Veber, P.S. Anderson, and R.M. Freidinger, Proc. Natl. Acad. Sci. U.S.A., 83, 4918-4922 (1986).

15. M.E. Van Dort, B.J. Cliax, D.L. Gildersleeve, P.S. Sherman, K.C. Rosenspire, A.B. Young, L. Joung, D.M. Wieland, J. Med. Chem., 31, 2081-2086 (1988).

16. (a). R.E. Olson, U.S. Pat., PCT int. Appl., WO 98/28268, 256pp (2000) (b). D. Seiffert, J.D. Bardley, C.M. Rominger, D.H. Rominger, F. Yang, Jr.J.E. Meredith, Q. Wang, A.H Roach, L.A. Thompson, S.M. Spitz, J.N. Higaki, S.R. Parakash, A.P. Combs, R.A. Copeland, S.P. Arneric, P. R. Hartig, D.W. Robertson, B. Cordell, A.M. Stern, R.E. Olson, and R. Zaczeg, The Journal of Biological Chemistry, 275, 34086-34091 (2000).

17. H.A. Dondas, R. Grigg, M. Thornton-Pett, Tetrahedron, 52, 13455-13466 (1996).

18. H.A. Dondas, S. Sonmez, Ileterocylic Comminications, 9, 23-30 (2003).

19. H.A. Dondas, J. Duraisigh.um, R. Grigg, W.S. MacLachlan, D.T. MacPherson, M. Thornton-Pett, V. Sridharan, S. Suganthan, Tetrahedron, 56, 4063-4070 (2000).

20. H.A. Dondas, PhD Thesis, Leeds University, Leeds-UK (1997).

21. H.A. Dondas, R. Grigg, C. Killner, Tetrahedron, 59, 8481-8487, (2003).

22. H.A. Dondas, R. Grigg, unjublished results.

\section{Received on November 28, 2003.}


